\title{
ONLINE TRIGGER SYSTEM TO SELECT PARTICLES GENERATED IN HIGH MULTIPLICITY ENVIRONMENTS
}

\author{
D. U. J. SONNADARA ${ }^{1+}$, W. E. CLELAND ${ }^{2}$ and S. A. VOLOSHIN ${ }^{3}$ \\ ${ }^{1}$ Department of Physics, University of Colombo, Colombo 3 \\ ${ }^{2}$ Department of Physics, University of Pittsburgh, Pittsburgh, PA 15260, USA \\ "Department of Physics \& Astronomy, Wane State University, Detroit, MI 48201, USA
}

(Received: 24 April 2000 ; accepted: 06 September 2000)

\begin{abstract}
An online hardware/software trigger system which is capable of tracking and identifying particles generated in high multiplicity environments was built and implemented. The hardware portion of the trigger generates the momentum of each track, while the software portion of the trigger finds the time of flight, permitting the particle identification. The electronic module developed for track recognition, based on 3000 series Xilinx Field Programmable Gate Arrays, were successful in recognising up to 2048 tracks in a $6.4 \mathrm{~ms}$ time interval. The details of: the trigger development and the online monituring of the trigger performance are presented.
\end{abstract}

Keywords: Field Programmab]e Gate Array (FPGA), particle triggers, multiplicity.

\section{INTRODUCTION}

Over the last few years, the central collision program of the E814/E877 collaboration' was heavily involved in addressing physics topics such as low $\mathrm{P}_{\mathrm{T}}$ enhancement, antiproton production and kaon interferometry. A major problem in this kind of analysis is to obtain enough statistics for each type of particle while keeping the background as low as possible. In the June 1990 and July 1991 runs (made with a ${ }^{2} \mathrm{Si}$ beam), a trigger in which the time of flight of particles reaching the downstream end of the spectrometer (roughly $30 \mathrm{~m}$ from the target) was at least 3 ns longer than that of the velocity of light particles, was used to enhance the data sample to study antiprotons. With this trigger, after removing the background, we were able to extract a few hundred antiprotons. However, the scintillator wall, which was used in extracting the time of flight for this trigger had granularity too coarse for Au-initiated collisions, for which this trigger was developed. Also, it was located too far downstream for efficient identification of kaons.

During the $1993 \mathrm{Au}$ run four multi-wire proportional chambers (MWPC) each with $0.5 \mathrm{~cm}$ wire spacing was added to the spectrometer to improve the tracking efficiency in the experiment. This was necessary since the pattern recognition became intractable when $10-15$ particles passed through the two tracking chambers in the spectrometer simultaneously. In addition, a new hodescope (TOFU)

* corresponding author 
with slat widths between 1 and $2 \mathrm{~cm}$ and good timing properties was installed in the upstream region ( $12 \mathrm{~m}$ from the target) of the experiment to improve the measurement of the time of flight of the particles. New phototubes with excellent timing were also installed in the beam scintillation counters to improve the measurement of the start time. We achieved roughly 80 ps resolution for the time of flight of particles with this new system. However, since the new scintillator wall was installed closer to the target to improve the efficiency for detecting kaons, it was more difficult for it to be included in a hardware trigger. Therefore, a new way of solving this problem in triggering rare event types was needed.

To satisfy this requirement, a trigger was developed that could track particles and reconstruct the momentum online. By combining this trigger with the precise timing information extracted from the new TOFU counters in software, it is possible to make a third level decision which includes particle identification. In this paper we discuss the various stages of development of this hardware/software trigger and online studies of the performance of the final system.

\section{METHODS AND MATERIALS}

A detailed description of the detectors used in the E814/E877 spectrometer is given elsewhere ${ }^{2,3}$ and hence only the necessary details are given in this paper. The detector layout basically consists of a set of scintillation counters to generate a beam trigger, a set of calorimeters to measure the energy of the particles, three tracking chambers and four multi-wire proportional chambers to calculate the momentum of particles and a set of scintillation hodoscopes to measure the time of flight and charge of the particles.

Preliminary tests of the tracking trigger electronics were carried out in the run of 1993. In the fall of 1994, $1 / 3$ of the full system was implemented and tested and in the fall of 1995, the full system was installed and run successfully in parallel with the normal data taking.

The instrumented region in the MWPC units used in the tracking trigger tests in the fall of 1994 is shown as the shaded region of Figure 1. Signals from the MWPC units were fed into a set of PCOS latching modules, which stored the data for readout through the PCOS controller, which in turn was used in the offline analysis. The additional 'prompt OR' output signals were used from the PCOS latching units consisting of an $\mathrm{OR}$ of two neighbouring wires (this configuration gives us roughly $1 \mathrm{~cm}$ readout spacing). These signals were fed into another set of electronic units (Latch \& Multiplexing units) mounted on a rack close to the target. These data were latched with the beam triggers and stored in the L\&M units until a pre-trigger followed by the level one trigger occurs. Once the level one trigger was issued, a signal was sent from the counting house to the L\&M units which in turn causes them to transmit the MWPC data to the counting house through a serial line to a set of Programmable CAMAC Xilinx (PCX) modules. 
The general-purpose PCX modules, developed at the University of Pittsburgh, are based on the 3000 series Xilinx Field Programmable Gate Arrays. They are capable of performing a wide variety of logical operations on two 16-bit input data words by storing the data on four 64-bit internal data registers and write the results to a single 16-bit output data word. Since only two 16-bit inputs are available, a total of 8 clock cycles are needed to load the MWPC data to the PCX data registers. The introduction of the L\&M between the PCOS units and the PCX units accomplished two important tasks: (a) intermediate storage of the data until the level one trigger is available and (b) orderly transmission of the data into PCX registers.

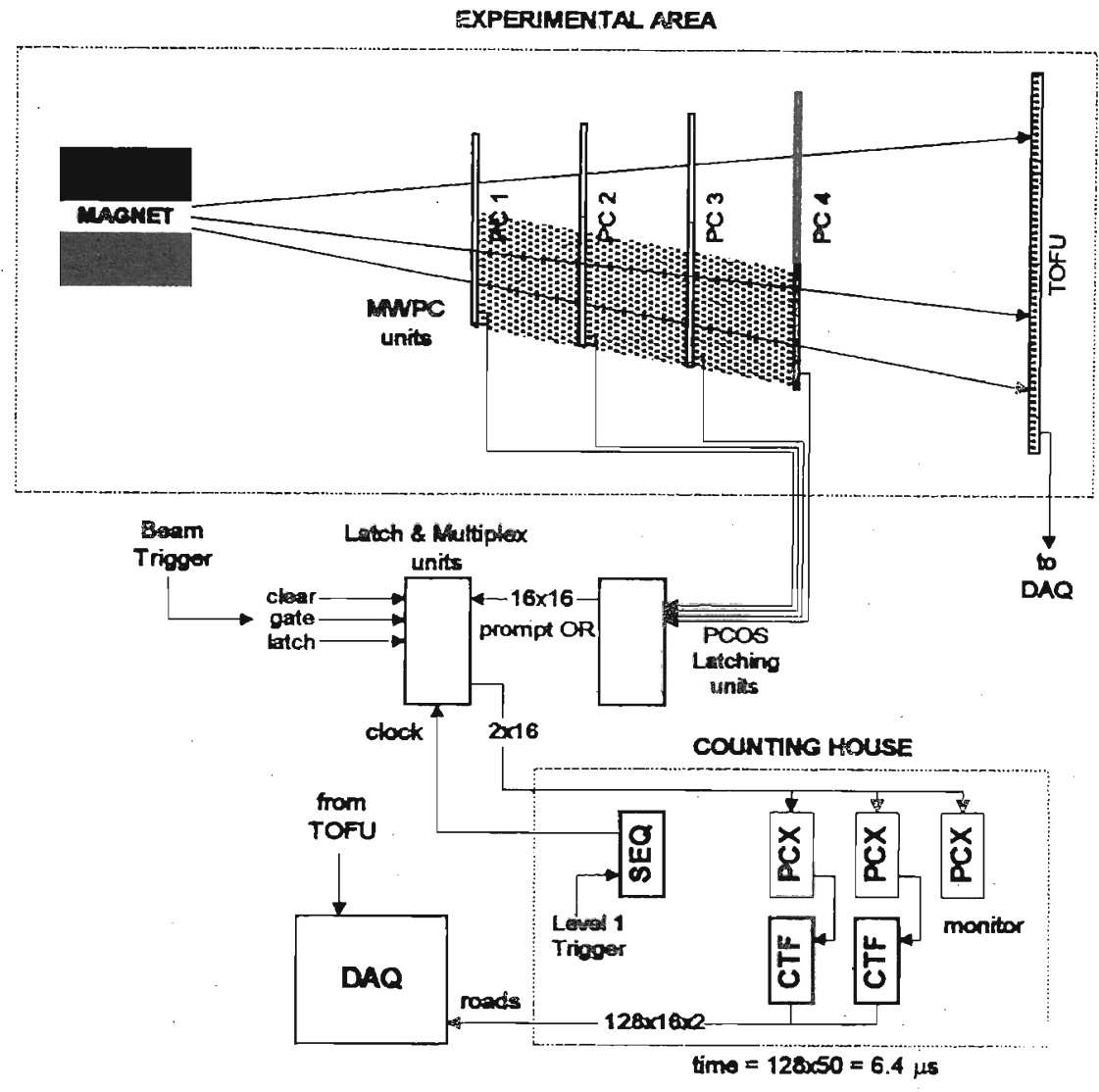

Figure 1: Diagram of the downstream region of the E814/E877 spectrometer showing the MWPC units. The shaded region is the area instrumented for the 1994 test of the tracking trigger.

To generate the possible track patterns, which should be recognised by the PCX units, the Heavy Ion Collision Simulation code (RQMD ${ }^{4,5}$ was used to generate the tracks in E814/877 spectrometer acceptance for the intended magnetic field 
settings. Each track pattern was defined in terms of the offset in readout spacings in the last three MWPC units with respect to the first MWPC unit. Due to the large number of possible track patterns and the limitations in the available time to analyse an event, all tracks passing through the same locations in the first and the last MWPC units were grouped as one, and defined as roads. In principle, each road can have a maximum of up to 5 different tracks since in this geometry, at least 3 out of 4 hits lie in the line formed by the first and the last MWPC units.

The PCX modules were programmed to identify the roads through coincidence logic, and the translation invariance of the roads was exploited by shifting the bit patterns in the PCX data registers. For the configuration used in the 1994 run, each PCX module was programmed to recognise up to 32 different roads for each of the 64 bit shifts, yielding a total track recognition capability of 2048 patterns per PCX. The shift clock driving the PCX was run at $20 \mathrm{MHz}$, and hence the track recognition step required $6.4 \mu \mathrm{s}$. In this test $3 \mathrm{PCX}$ modules were used; 2 for pattern recognition (one for tracks with positive gradient and the other for tracks with negative gradient) and the remaining one to monitor the operation and the performance of the PCOS and L\&M units. The output of the pattern recognition results was stored in two LeCroy Data Stack modules. The data stacks and the monitoring PCX were read by the data acquisition system and become part of the event record, permitting us to study the tracking trigger performance offline as well as online.
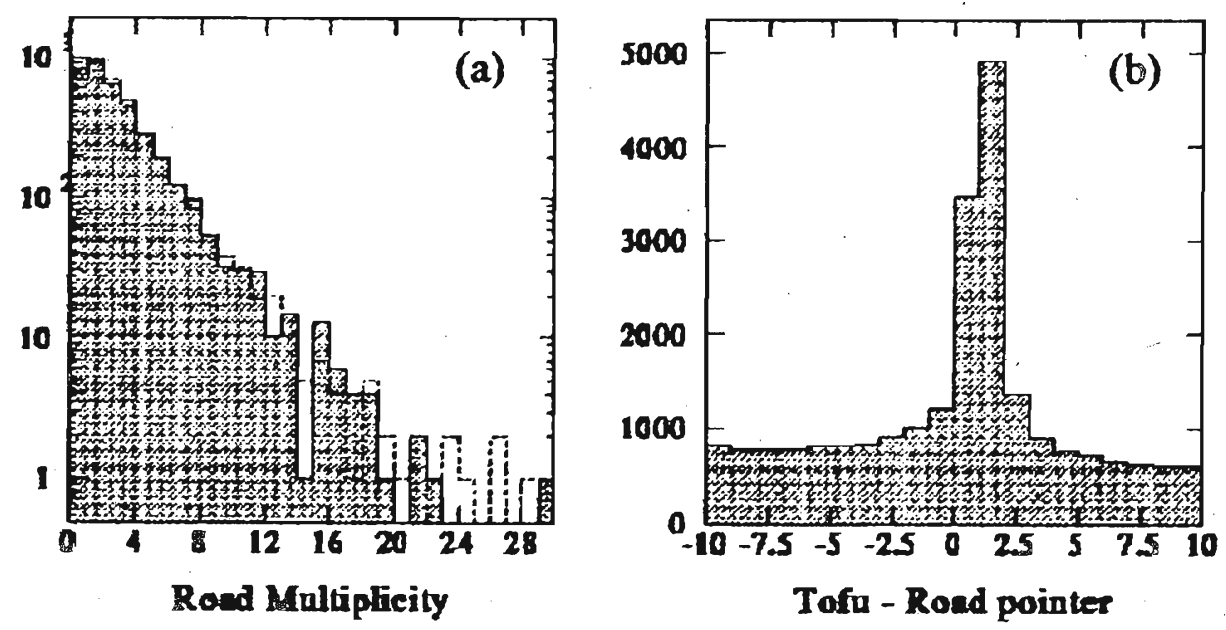

Figure 2: (a) Multiplicity of tracks found by the tracking trigger hardware during the 1994 run. (b) Correspondence between the track pointers and TOFU counters containing actual hits. 
Figure 2a shows the multiplicity of the roads (solid line) for the region which was instrumented. In the same figure the multiplicity with TOFU coincidence is shown (dashed line). There is no significant reduction with TOFU coincidence, which indicates that most of the tracks are real. Figure $2 \mathrm{~b}$ shows the coincidence between the TOFU counters which are supposed to be hit by passing particles as predicted by the track pointers and the counters containing the actual hits. It is clear from this plot that track pointers are sufficiently accurate to narrow the window to within 2 TOFU counters. Accidental hits generate a background of about $20 \%$, which are responsible for the flat distribution in this plot.

To understand the performance of the tracking trigger, a third level trigger logic was implemented at the software level. In the third level, the time of flight counter number and the momentum were obtained by a lookup table based on the road number, and only the time of flight itself needed to be calculated. Similar calculations were applied in an offline analysis to extract the time of flight corresponding to a given road number to study the online trigger operation.

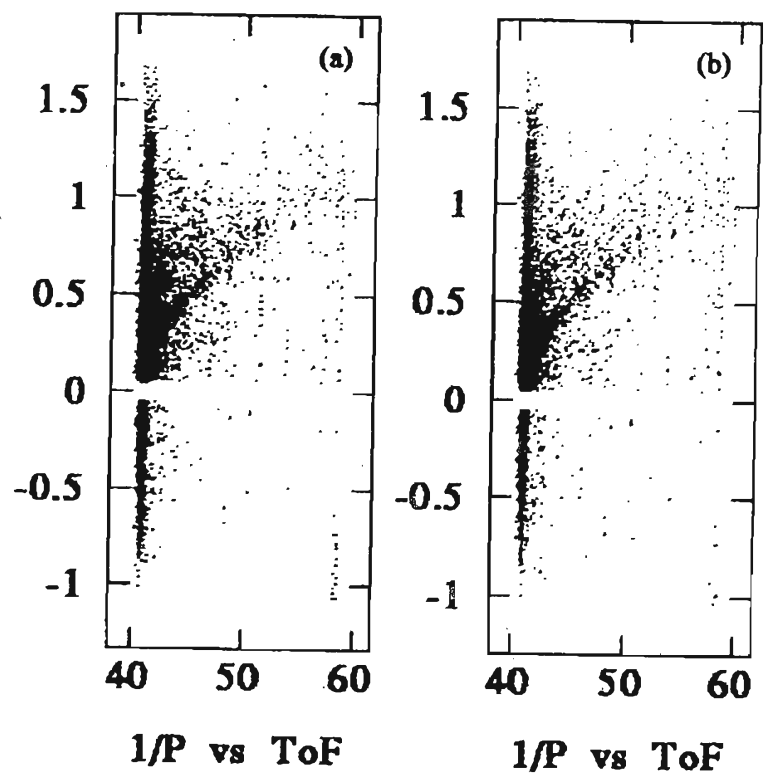

Figure 3: Plot of inverse momentum (1/p) vs. time of flight, which was used for the identification of particles. (a) Data produced by the tracking trigger hardware. (b) Data produced by offline track reconstruction, which simulates the hardware. 
Figure $3 \mathrm{a}$ shows the particle ID plot ( $1 / \mathrm{P}$ vs. time of flight) reconstructed from the online hardware trigger for real data. In this plot the mass bands for protons and pions were identified. However, the kaon mass band was populated by false tracks. Figure $3 \mathrm{~b}$ shows the same plot generated in offline by simulating the hardware logic. A very good agreement was seen, indicating that the PCX track-finding logic and the tracking trigger hardware was performing as expected.

\section{RESULTS AND DISCUSSION}

To understand the performance of the full system before implementation, the Monte Carlo simulation as well as real data taken in June 1994 was used.

For the Monte Carlo simulation, the events generated by RQMD were used and crated the hit distributions that could be observed in the MWPC and the TOF counters on an event by event basis. A resolution of $100 \mathrm{ps,} \mathrm{which} \mathrm{could} \mathrm{be} \mathrm{achieved}$ at the level of the third level trigger, was assumed for the TOF counters. Since 3 PCX units were needed to cover the entire area in the spectrometer where the particle tracks passed through, 3 logical regions were defined and MWPC data from each region was fed into a single PCX. These regions were defined with special attention to minimise the loss in tracks crossing different regions. The calculation of the TOF and the track reconstruction were done by the same technique envisioned for the third level trigger. If two or more particles go through the same TOF counter, only the time from the fastest particle was used.

For the real data, the PCX track reconstruction logic was applied at the software level (for the hits recorded by MWPC units) to generate roads and the same steps discussed in the previous paragraph were followed.

The electronics needed to set up a tracking trigger covering all 3 regions consists of 3 L\&M units, 9 PCX modules ( 6 for pattern recognition and 3 for monitoring) and 6 CTF (CAMAC transversal filter) modules. The CTF modules, which were also developed at the University of Pittsburgh, are capable of performing digital arithmetic functions, but in this case they were used as data stacks. The CTF modules have two advantages over standard LeCroy data stacks since they (i) can be programmed to suppress the data words containing only zeros (ii) cost a factor of two below the LeCroy data stacks. To control the operation of the tracking trigger timing sequence, a Sequencer module, also constructed at the University of Pittsburgh, was used.

Figure $4 \mathrm{a}$ indicates the expected track efficiency for the full system as a function of the momentum of the particles. The open histogram shows the reconstructed tracks and shaded histogram shows the tracks that are missed. Tracks that are going into the beam region are not considered here. An overall efficiency of above $95 \%$ was observed. 

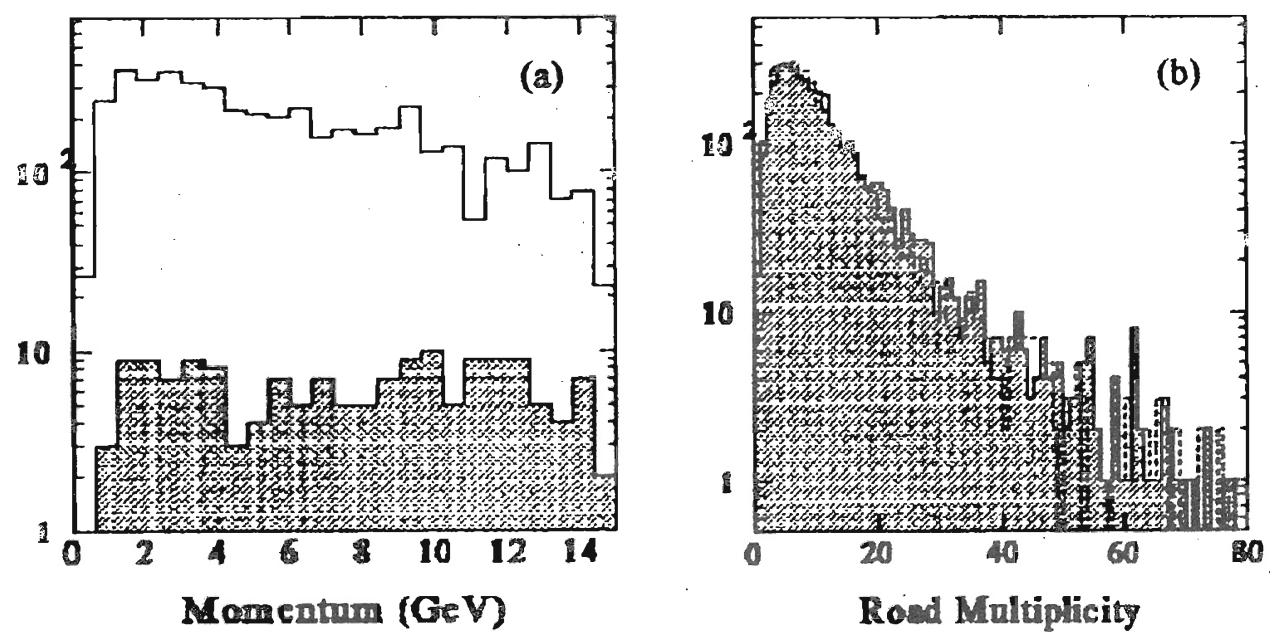

Figure 4: Track efficiency as a function of momentum and multiplicity. (a) shaded histogram: tracks not found by the tracking hardware. (b) Dashed line: track multiplicity when TOF counter is required.

Figure 4b shows the track multiplicity from only the MWPC data (solid) and MWPC plus TOFU coincidence (dashed). These data indicate that there is no significant reduction by including the TOFU coincidence. At one stage it was considered to include TOFU as an additional plane in the tracking trigger logic. This option was later rejected since it unduly complicated the logic used in the trigger design and was justified by the results shown in Figure 4b. However our results indicate that the third level trigger needs to perform at least 20-30 TOFU time of flight reconstructions to extract the mass information per event on average.

Figure 5 shows the Monte Carlo generated particle ID plot for true tracks and false tracks. For true tracks the resolution was adequate to identify protons, pions and kaons. However, the false tracks (which are accidental coincidences of hits of two or more real tracks) were not uniformly distributed but rather formed a plot that is complimentary to the one for real tracks. When the plots were superimposed (see Figure 6b) the resulting distribution appeared to have poor resolution, but for real tracks, the true resolution was still which is shown in Figure 5a.

Figure 6 a shows the same plot for real data, which looks somewhat worse than the Monte Carlo. One reason for this is the existence of multiple tracks for a given time of flight counter (they are responsible for the vertical lines in the figure) which can be generated by noise in the TOFU counters. 

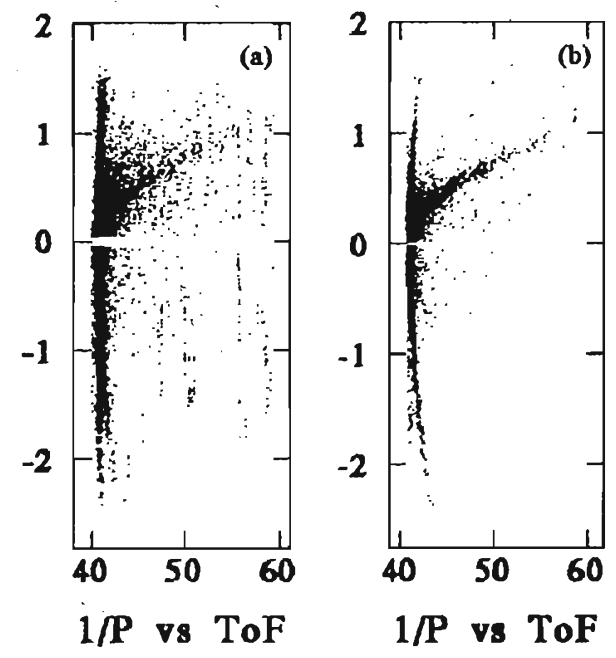

Figure 5: Particle ID plots from the Monte Carlo simulation (a) true tracks (b) false tracks
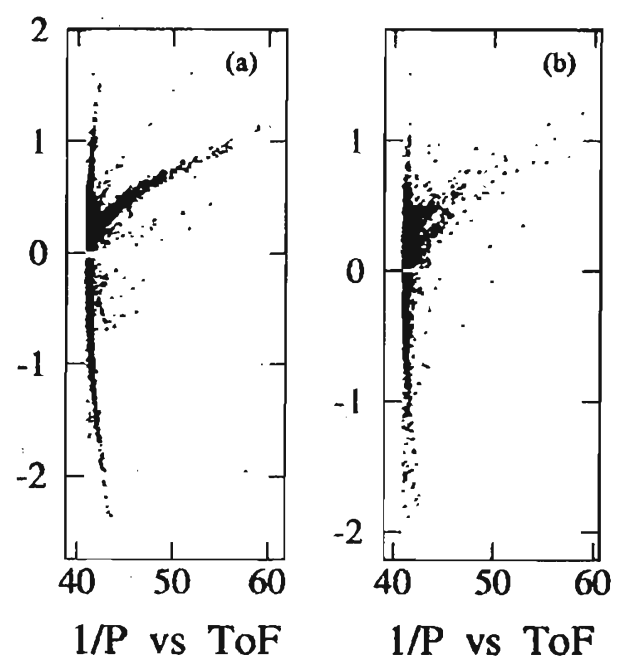

Figure 6: Particle ID plots for (a) real data (b) Monte Carlo simulation. The Monte Carlo simulation is the sum of the two plots shown in Figure 5. 


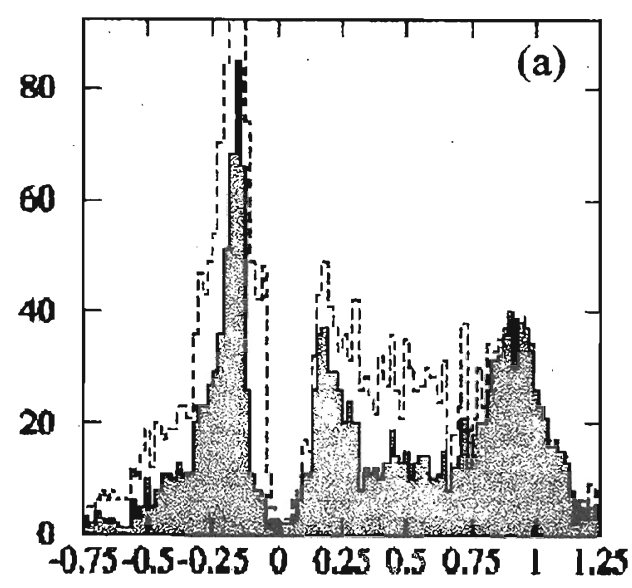

Mass

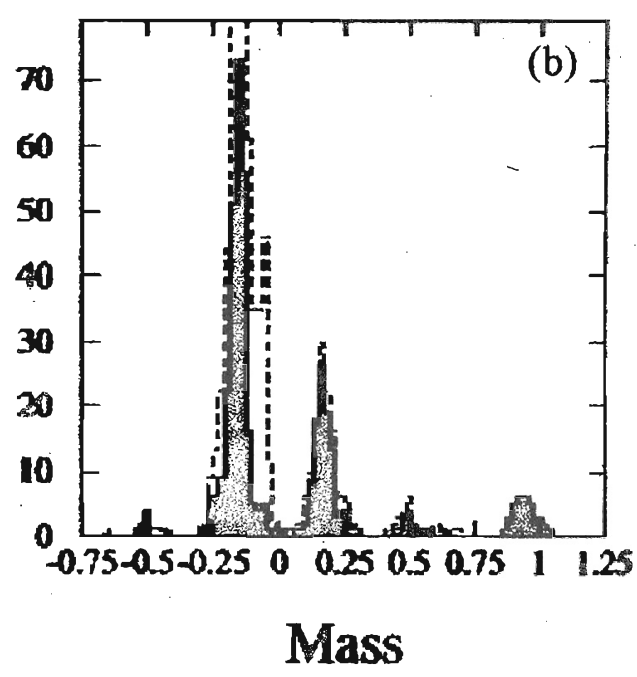

Figure 7: Monte Carlo mass distribution simulated for the third level trigger. In both plots, the open histogram is the distribution for all tracks and the shaded histogram is for true tracks only. (a) Momentum is required to be less than 7 $\mathrm{GeV} / \mathrm{c}$. (b) Momentum is required to be less than $2 \mathrm{GeV} / \mathrm{c}$.

Figure 7 shows the reconstructed mass for the Monte Carlo data for two momentum cuts. Each figure shows the mass reconstruction for all tracks (open histogram) and only true tracks (shaded histogram). They show that although false tracks create a significant background, we can nevertheless apply trigger cuts on the mass since we have a higher efficiency in selecting true tracks. For the low momentum region, false tracks do not have an effect.

\section{Online Monitoring}

The tracking trigger was fully operational during the final (1995) run of Experiment 877. The third level tracking trigger logic could select events using three different physics criteria:

1. events which have at least two negative particles (mostly pions),

2. events with at least one negative relatively slow (non pion) particle, and

3. events with a pair of non-pion particles.

The selections are made according to the occupancy of a selected region in the particle ID plot (inverse momentum versus time-of-flight). The third level trigger logic allowed the application of independent downscaling to each of the triggers. 
The monitoring that was developed allowed us to estimate the trigger rate online (the trigger efficiency is close to $100 \%$ for the logic used). Due to a dramatic improvement in the data tape writing speed (see below) there was no need to apply the tracking trigger for the very central events; this trigger was used most of the run to process semi-central (PCAL/TCAL level 2) events (see below). The trigger rate for two negative pions for semi-central events was somewhat higher than expected (about 50-60\%) and was used during a two week running period. The trigger for at least one non-pion particle was used in most of the run without downscaling. The trigger rate reduction for this condition was about $30 \%$. In this case there was no need to trigger on two negative non-pion particles and it was never used as making a decision (but it was used for monitoring purposes).

\section{Tracking trigger monitor page 1}

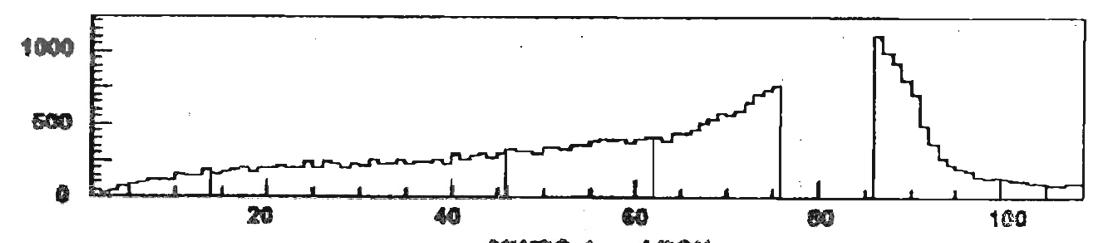

arenc-1 and pex
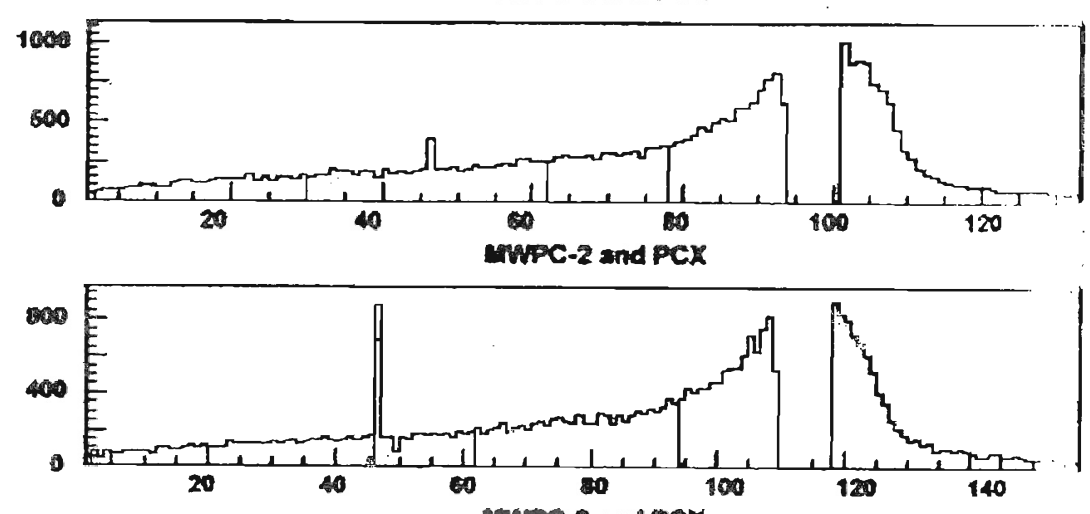

unclas and pex

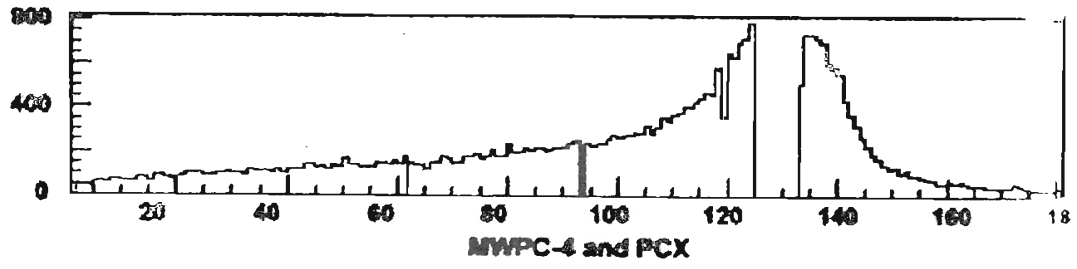

Figure 8: Comparison between the hit distribution in MWPC units with the corresponding hit distribution in PCE modules

As described above, the tracking trigger decisions are based on the signals which were read from MWPCs using the prompt "OR" output signals from the PCOS 
latch cards. These data were latched externally and transmitted through a multiplexer into the PCX card, a CAMAC card that contains a large Xilinx Field Programmable Gate Array chip. To verify the integrity of the input data used by the tracking trigger, the online information stored in the PCX was compared with that read from the normal CAMAC readout of the PCOS system. On the tracking trigger monitor page 1 (Figure 8), the hit distributions for each MWPC with hit distributions derived from PCXs were compared. This monitor page was also used to monitor the performance of MWPCs and related electronics.

Tracking trigger monitor page 2 (Figure 9) was devoted to monitoring of pattern recognition and track reconstruction (CTF performance). Using this page we monitor the pointing of tracks (reconstructed at CTF from PCXs information) pointing to the TOFU counters. From our calculation it followed that the pointing accuracy should correspond to about 2 TOFU counters. We monitor track pointing for each of 3 covered regions for positive and negative shifts separately. On this page we also monitor the total number of non zero CTF words which is strongly correlated with the total number of reconstructed tracks and was used in the trigger logic to reject events with high track multiplicity. The total multiplicity of "positive" and "negative" tracks were also monitored for the purpose of defining. the proper cuts in the third level trigger.

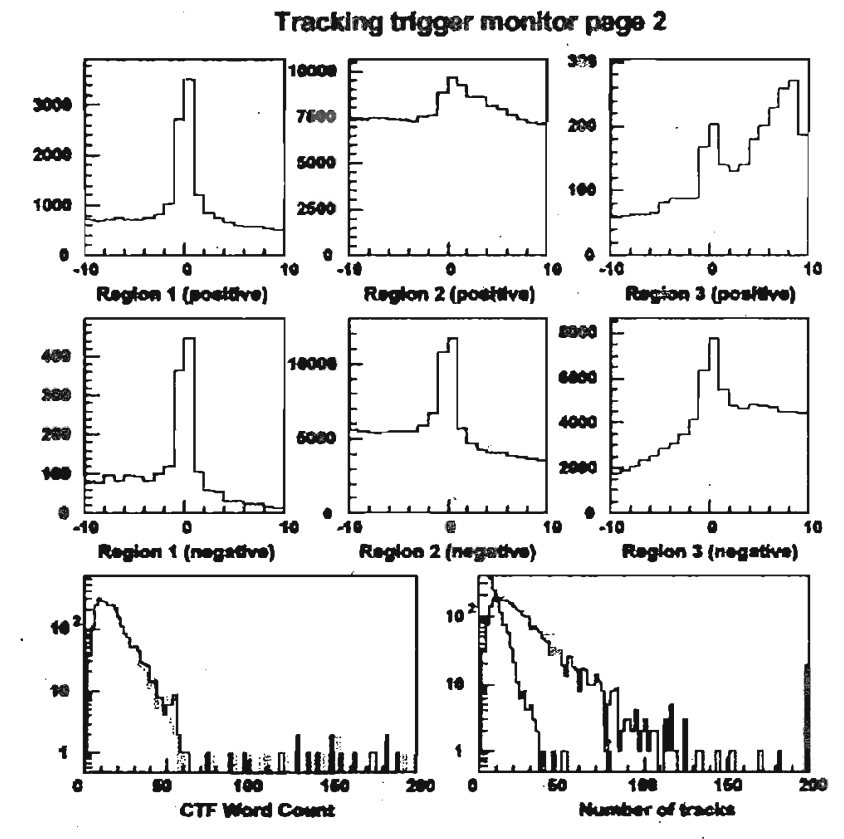

Figure 9: Pointing accuracy of the track reconstruction and TOFU coincidences 
The third level trigger monitor page (see Figure 10) was used for monitoring of the "physics" of the tracking trigger - the process of making decision of accepting the event. On this page the trigger rates and downscaling factors for each of used selection criteria (and downscaling for events without selection criteria applied) were first monitored and combined with trigger rate. Then the online particle ID plot was drawn. On the same plot we draw regions used by the third level trigger logic for identification of a hit as pion or non pion. That permits some judgment on momentum and time-of-flight reconstruction. On the same page we monitor also TOFU calibration constants (TO values) used for particle ID. This plot shows the mean difference between measured and calculated time of arrival of pions, and it is very sensitive to drifts in the constants associated with the time of flight system. Thus by examining this plot, the shift crew was able to determine if it was necessary to update the TOF constants.

\section{Tracking trigger monitor page 3}
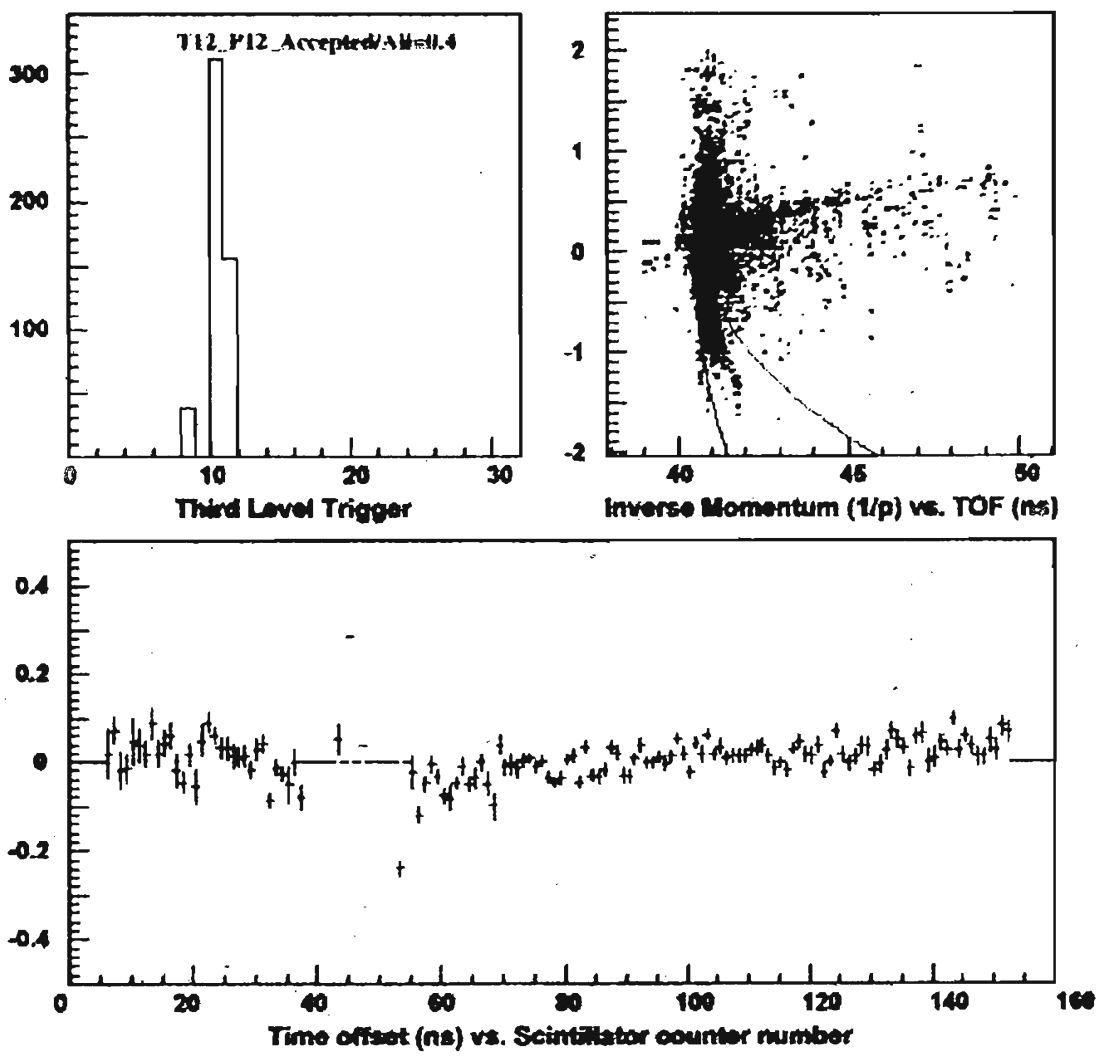

Figure 10: Monitoring of trigger downscaling, online particle identification and t-zero 
A hardware/software trigger built, installed and implemented at the third trigger level successfully identified and counted the particles (pions or non-pions). The hardware portion of the trigger finds the slope and intercept (and hence momentum) of each track, and in software the time of flight information can be obtained, permitting the mass of the track to be calculated. The trigger performed correctly, making it possible to accept events of a particular topology. Although a part of the data was taken with this trigger, a parallel development in the data acquisition permitted a dramatic increase in tape writing ability, and hence the actual need for the trigger in analysing data was minimal. For this reason, physics studies from this set of data were carried out using unbiased data taken without this trigger, so that detailed studies of the trigger efficiency and the biases it introduces were unnecessary. Thus the most complete studies of this trigger were those performed during the online monitoring in making sure that the correct events were written to tapes, the work that is presented here.

\section{Acknowledgement}

This work was supported in part by the U.S. Department of Energy, Grant No. DEFG02 87ER40363.

\section{References}

1. Lissauer D. et al. (1985). Study of extreme peripheral collisions and of the transition from peripheral to central collisions in reactions induced by relativistic-heavy ions, Proposal 814 submitted to the AGS program committee, Brookhaven National Laboratory, New York.

2. Barrettee J. et al., E814 Collaboration (1995). Electromagnetic dissociation of relativistic 28Si. Physical Review C 51, 865

3. Barrettee J. et al., E877 Collaboration (1995). Charged particle pseudorapidity distributions in $\mathrm{Au}+\mathrm{Al}, \mathrm{CU}, \mathrm{Au}$, and $\mathrm{U}$ collisions at $10.8 \mathrm{~A} . \mathrm{GeV} / \mathrm{c}$. Physical Review C 51: 3309

4. Sorge H., Stoker H. \& Greiner W. (1989). Poincare invarient Hamiltonian dynamics: modelling multi-hadronic interactions in a phase space approach. Annals of Physics (NY) 192: 266

5. Jahns A., Sorge H., Stocker H. \& Greiner W. (1992). Strong antiproton suppression in relativistic nuclear collisions. Zeitschrift $\ddot{F} u r$ Physik A 341: 243 